\title{
Apport lipidique et prise de poids
}

\author{
Aspects quantitatifs - Un débat
}

Jean-Michel LECERF

Service de nutrition,

Institut Pasteur de Lille BP 245 -

59019 Lille Cedex

<jean-michel.lecerf@pasteur-lille.fr>

Service de médecine interne -

CHRU de Lille

<jean-michel.lecerf@pasteur-lille.fr>

\begin{abstract}
From a quantitative point of view the role of dietary fat in weight gain apparently seems clear, but its accountability in obesity pathogenesis, for individuals or population, is not definitively established. This concern may be divided in four sections. On a physiopathological point of view, experimental data show that fats are able to increase energy density and food intake and to increase lipogenesis. On an epidemiological point of view the data are more conflicting. An higher fat intake was associated to the obesity epidemic, its decrease did not stopped it, and that suggest the role of others lifestyle patterns including physical activity. On a clinical point of view the studies are far from a consensus, but it is true that they focuse on the role of familial, education and genetic factors. But really a fat excess can sensitive the effect of inactivity. Total energy intakes seem a main issue. The fat reduction is not sufficient to induce a weight loss or to prevent a weight gain. We must act on each component of energy balance. In insulin-resistant subjects the fat reduction without weight-loss does not seem favourable on cardiovascular risk. Finally the total fat intakes cannot be considered as the cause of obesity but they may participate to the weight gain in some conditions. Qualitative aspects, and therefore fatty acids, must be also considered.
\end{abstract}

Key words: Fat intake, weight gain, obesity, lipogenesis, cardiovascular risk

\section{Introduction}

L'épidémie d'obésité qui concerne la plupart des pays du monde pose question. Elle conduit à la recherche des facteurs explicatifs, et si possible causaux, et donc responsables de cette inflation. Le coupable désigné est bien sûr I'alimentation, mais l'accusation n'est pas assez précise : les témoins à charge contre les lipides sont nombreux, en tout premier l'apport énergétique des lipides $(9 \mathrm{kcal} / \mathrm{g}$ ) car il dépasse celui des autres nutriments. Questions de lipides ou de calories? Dans ce cas, pourquoi pas aussi les glucides? À moins que le rapport lipides/glucides (les 2 nutriments étant toujours inversement corrélés) soit en cause ? Mais plus que les lipides ne serait-ce pas la densité énergétique qui déterminerait la surconsommation? Mais s'il s'agit de surconsommation, c'est par rapport à une dépense, et donc la balance entrées/sorties ne serait-elle pas d'avantage impliquée dans ce bilan excédentaire ? À moins que ce soit les voies énergétiques de stockage ou d'oxydation qui expliquent la prise de poids ? La prise de poids est d'ailleurs peut-être plus discriminante de l'obésité qui, elle, peut être un état statique, résultant lui-même d'une dynamique qu'il conviendrait de prendre en compte. Mais toute la population n'est pas obèse, et de même parmi les enfants tous ne prennent pas (encore) du poids: quelle est donc l'interaction entre l'apport lipidique (ou énergétique) et la susceptibilité individuelle, elle-même liée essentiellement à la prédisposition génétique? À moins que les lipides ne soient pas tous égaux entre eux, leur nature pouvant l'emporter sur leur quantité?

Nous tenterons de répondre à ces questions avec des données expérimentales, épidémiologiques et cliniques.

\section{Données expérimentales}

Les lipides ont « ce qu'il faut » pour faire prendre du poids. D'abord, parce que bien qu'ils ne contribuent que pour 37 à $39 \%$ aux apports énergétiques (contre 45 à $55 \%$ pour les glucides) ils fournissent, au gramme, plus de calories que les glucides (9 vs 4). D'autres arguments doivent être avancés cependant.

D'une part, la forme de stockage de l'énergie chez les mammifères se fait quasi exclusivement sous forme de triglycérides au niveau du tissu adipeux et ceci de façon expansible (plus de 100000 kcalories) ; d'autre part, et surtout, les voies métaboliques qu'ils empruntent sont davantage orientées vers le stockage que vers l'oxydation, alors que le stockage des glucides, sous forme de glycogène, est très limité (1 000 kcalories). En effet, de nombreuses études [1-3] ont montré que dans des conditions de manipulation alimentaire, avec suralimentation sur le court terme, la surconsommation de lipides induit une faible oxydation des lipides, et donc conduit au stockage, alors qu'une suralimentation glucidique équivalente (en énergie) induit une forte oxydation glucidique (mais elle réduit l'oxydation des lipides). De façon caricaturale on pourrait dire qu'un excès de graisses favori- sera le stockage des lipides, mais qu'un excès de glucides fera stocker les lipides alimentaires ; ou encore qu'une surconsommation de bonbons (!) fera stocker la mayonnaise que I'on mange si, à cause des glucides alimentaires, l'apport énergétique dépasse les dépenses ! Les travaux de l'équipe de Prentice sont très éloquents à cet égard [4].

De même, l'incidence du gain de poids de plus de $5 \mathrm{~kg}$ est plus élevée lorsque le quotient respiratoire est plus haut, témoin d'une oxydation glucidique accrue et donc d'une oxydation lipidique moindre et donc d'un stockage de lipides accru [5]. Mais l'oxydation lipidique croît avec la masse grasse, ce qui est un élément contributif à la résistance à l'amaigrissement [6] (tandis que l'oxydation glucidique décroît avec la masse grasse). Le post-obèse oxyde moins les lipides que le sujet témoin, surtout lorsque l'apport lipidique croît, ce qui témoigne aussi d'une « inégalité » conduisant l'obèse à une capacité importante à regrossir si I'apport lipidique s'élève [7]. Cette interaction entre apport lipidique et susceptibilité génétique est à rapprocher d'une étude montrant que lorsque l'apport lipidique augmente, seuls ceux qui ont des parents obèses et un surpoids initial prennent du poids, pour un seuil lipidique donné [8] (figure 1). L'équipe de Ravussin a montré que pour un seuil lipidique de $30 \%$ la balance lipidique était positive chez le postobèse alors qu'elle est négative chez le sujet témoin [9].

Les lipides sont aussi les nutriments qui induisent le plus de palatabilité (composante affec- 
tive alimentaire) [10], ce qui explique que les préférences alimentaires soient corrélées à la densité énergétique, elle-même très fortement corrélée à la teneur en lipides des aliments. Or I'on consomme ce que l'on préfère ; ceci est vrai pour les aliments gras comme pour les aliments maigres [11] (mais l'on préfère plus souvent les aliments gras). II a d'ailleurs été montré une relation entre la réponse hédonique maximum et la prise de poids [12] ; par contre, il n'y a pas d'effet du changement du poids sur le changement de réponse hédonique [12].

Les obèses pourraient donc surconsommer des aliments lipidiques parce qu'ils ont initialement des préférences accrues pour les aliments gras. Dans une étude cas-témoins chez des enfants obèses et de poids normal, nous avions montré il y a quelques années qu'il n'y avait pas de différence de préférences entre enfants obèses ou non [13]. Drewnowski avait montré sur une échelle de préférence que les obèses avaient un score plus bas que les sujets témoins pour le gras, mais les obèses fluctuants ont une préférence alimentaire plus élevée pour les lipides [14]; or un obèse fluctuant est un obèse en dessous de son poids de consigne et donc en restriction car « au régime ». Le même auteur avait montré que seuls les obèses fluctuants ayant débuté tôt leur obésité avaient une préférence pour les lipides [14].

Une expérience menée par l'équipe de Rolls a démontré qu'en faisant varier la densité énergétique d'une alimentation on induisait expérimentalement un apport énergétique plus important, quoique le poids de nourriture ingérée fût le même [15]. De même la surali- mentation énergétique spontanée, ad libitum (en présence d'aliments hyperlipidiques), ainsi que le pourcentage de calories lipidiques sont corrélés à la masse grasse corporelle [16]. Mais il s'agit de conditions expérimentales.

Par contre, en situation réelle d'observation épidémiologique, la densité énergétique de I'alimentation globale n'est que peu corrélée à I'apport lipidique et bien qu'il existe une corrélation entre densité énergétique et apport énergétique total, il y a une relation inverse paradoxale entre densité énergétique et index de masse corporelle [17]. Ceci pourrait être dû à un biais relatif à la non-prise en compte des boissons, mais aussi au caractère dynamique de l'obésité, les obèses se restreignant; ou encore à la non-prise en compte d'autres facteurs : c'est le cas de l'inactivité physique. Dans un protocole expérimental avec 15 jours de régime isoénergétique hyperlipidique ou hyperglucidique, avec activité physique stable, suivis d'une journée d'inactivité, la balance lipidique est plus positive et plus souvent positive avec le régime « high fat » [18].

De plus, une suralimentation lipidique pendant 2 semaines induit une moindre dépense énergétique nocturne qu'une suralimentation glucidique [19]. Enfin, I'on sait que les lipides induisent une moindre thermogenèse postprandiale que les protéines ou les glucides si ceux-ci sont stockés sous forme de glycogène [20].

Un travail expérimental a, de plus, montré qu'un régime riche en lipides faisait non seulement prendre plus de masse grasse mais aussi moins de masse maigre qu'un régime riche en glucides [21], ce qui induit donc un moindre

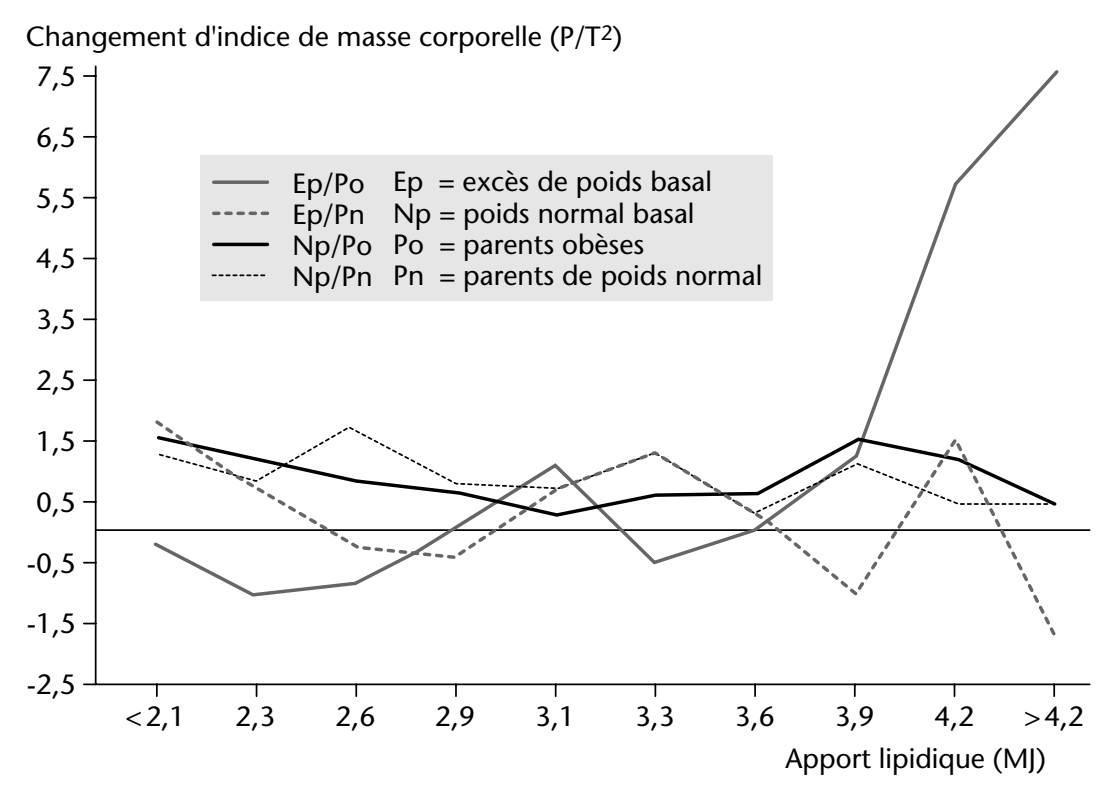

Figure 1. Changement d'indice de masse corporelle $\left(P / T^{2}\right)$. D'après Heitmann AJCN $1995 ; 61$ : 1213-7.

métabolisme basal: les «ingrédients» pour favoriser la survenue de l'obésité sont là. Ainsi, au total les lipides sont d'excellents substrats pour la lipogenèse et donc de très bons candidats pour la prise de poids.

\section{Les données épidémiologiques}

Là aussi l'évidence pourrait paraître éclatante. Ainsi par exemple l'obésité chez les jeunes recrues danoises entre 1935 et 1985 est croissante et parallèle à la courbe de l'apport lipidique (en pourcentage des apports énergétiques) [22]. Mais il ne s'agit que du rapprochement de données statistiques. La célèbre étude de migration Ni Hon San Study a bien mis en évidence le rôle de l'accroissement de l'apport lipidique dans l'élévation de l'indice de masse corporelle (IMC) mais cela ne peut être séparé des autres composantes du mode de vie [23]. L'étude des 7 pays confirme une corrélation entre le pourcentage de lipides dans la ration et le poids médian relatif des individus regroupés par pays : mais le regroupement par pays apporte un biais majeur [22]. D’autres données épidémiologiques écologiques mettant en relation les apports lipidiques calculés (inférieurs à $35 \%$ ) à partir des disponibilités alimentaires (FAO) dans 11 pays en voie de développement et la déviation du poids moyen de la population par rapport au poids standard montraient une corrélation positive [22]. Une autre étude écologique citée par Bray [25], avec dans un choix de 20 pays le pourcentage de lipides moyen dans chaque population et le pourcentage de sujets ayant un IMC de plus de $25 \mathrm{~kg} / \mathrm{m}^{2}$, retrouve la même corrélation mais le recueil des données via les disponibilités alimentaires est critiquable ; et la corrélation ne préjuge pas de la relation. Si I'on considère I'IMC moyen, l'étude MONICA avec 18 régions européennes [22] a mis en évidence chez les femmes une corrélation inverse avec le pourcentage de lipides dans la ration, et une absence de corrélation chez les hommes : mais la valeur de I'IMC moyen comme reflet de la situation d'un pays est critiquée. D'autre part chez les femmes occidentales le surpoids est associé à une tentative de réduction des apports énergétiques et/ou à une sousestimation (cf. infra), ce qui peut largement fausser les données. Aux extrêmes l'étude Leeds Fat Study a montré qu'en cas d'IMC supérieur à 30 , il y a 19 fois plus de sujets consommant une diététique très hyperlipidique (plus de $45 \%$ ) qu'une diététique moins lipidique (35\%) [23].

La fameuse étude transversale de Bolton-Smith montre une corrélation inverse entre apport en sucres et obésité (\%) et une corrélation positive entre les quintiles du rapport lipides/sucres et le 
pourcentage de sujets en surpoids et obèses étant accentué par le fait qu'il existe une relation inverse connue entre lipides et glucides [25]. Une autre étude britannique transversale menée par l'équipe de Blundell [26] a confirmé I'absence de corrélation entre sucres et IMC et I'existence d'une corrélation entre apport lipidique et IMC, mais en considérant les sousévaluateurs ( $40 \%$ des femmes et $27 \%$ des hommes, beaucoup plus nombreux si I'IMC est supérieur à 30) on passe, chez les femmes, $d^{\prime} u n e$ relation négative entre aliments gras sucrés et IMC en incluant les sous-évaluateurs, à une relation positive en les excluant.

Un autre paradoxe lipidique s'exprime dans ans sur deux périodes $[27,28]$ : alors que la prévalence du surpoids a augmenté de $32 \%$, I'apport lipidique a baissé de $10 \%$, et celui des calories a baissé de $6 \%$ en moyenne dans le même temps (figure 2). En Grande-Bretagne, Prentice [29] a attribué le même paradoxe à l'augmentation de la sédentarité mesurée par deux indicateurs: le nombre de voitures par habitants et le nombre d'heures de télévision.

II est évident que le style alimentaire et plus largement le style de vie jouent un rôle plus grand dans la survenue de l'obésité. Toutefois dans une revue de la littérature analysant 30 études et regroupant 3 styles alimentaires: 1) viandes, gras, sucreries ou aliments denses; 2) légumes, fruits, céréales complètes et aliments à teneur lipidique réduite ; 3) consommation élevée d'alcool. II apparaît que dans 10 études, le style 1 est associé positivement avec I'IMC/obésité, mais dans 4 études le style 1 est chez les hommes et chez les femmes [24]; ceci l'évolution de I'IMC aux Etats-Unis en quinze

associé négativement et dans 11 autres études il n'y a pas d'association [30].

La densité énergétique pourrait être un élément plus important dans le lien entre alimentation et prise de poids : or, quel que soit le pourcentage de lipides ( $\leq 30 \%$ ou $>$ à $30 \%$ ) elle est inversement corrélée au nombre quotidien de portions de fruits et de légumes. De sorte qu'un régime plus riche en lipides (> $30 \%)$ n'est pas associé à une plus forte prévalence de l'obésité lorsque l'apport en fruits et légumes est élevé, alors que l'obésité est plus fréquente lorsque l'apport en fruits et légumes est moindre, même en cas de faible apport lipidique $(<30 \%)$ [31] (figure 3 ). Ceci peut être un élément de plus expliquant la faible corrélation, quoique positive, entre apport lipidique total et gain de poids dans l'étude prospective (8 ans) des infirmières américaines. Cependant, l'association était significativement plus forte, quel que soit le type de lipides, parmi les femmes en surpoids que parmi les femmes minces [32], ce qui est en faveur du rôle aggravant d'une prédisposition.

\section{Les données cliniques}

Les études cliniques apportent un autre éclairage.

Compte tenu du caractère dynamique de l'obésité et des effets "perturbateurs", de la restriction alimentaire d'une part et de la résistance à l'amaigrissement d'autre part, il est particulièrement intéressant d'analyser les études chez l'enfant encore peu atteint par ces phénomènes. La sous-évaluation devrait cependant être également prise en compte,

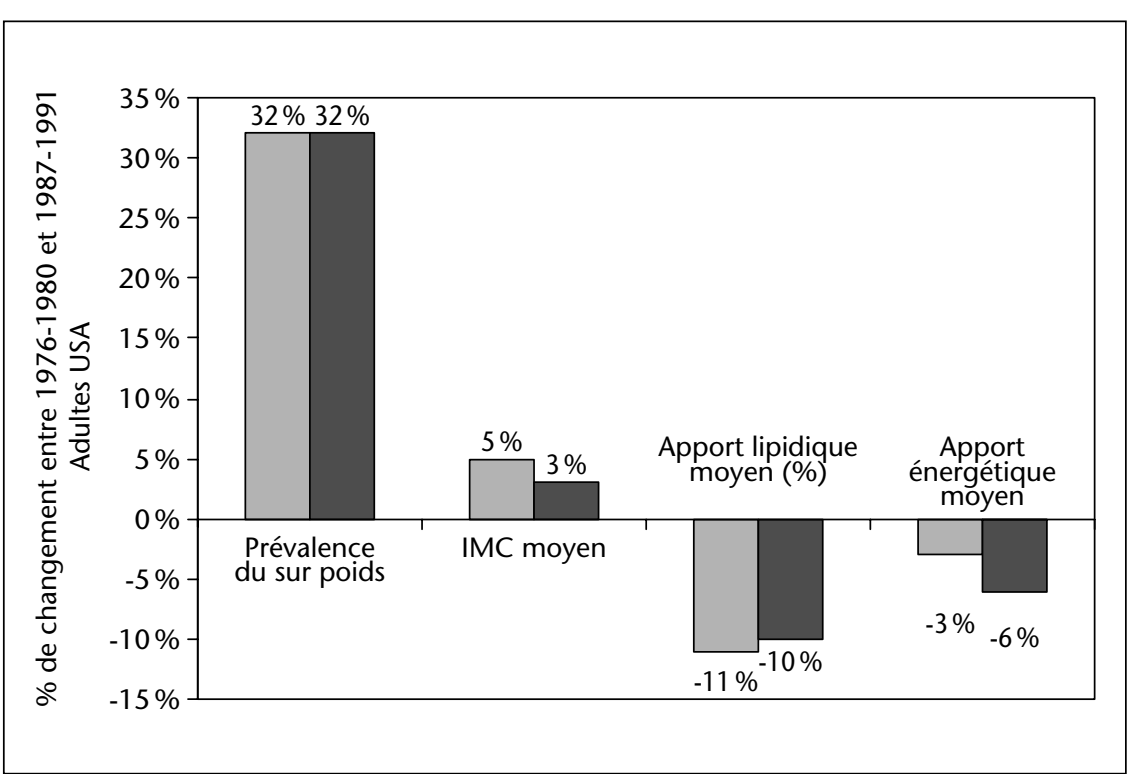

Figure 2. Evolution du surpoids et de I'IMC aux USA sur 15 ans et apports nutritionnels. D'après Heini, Am I Med $1997 ; 102: 259-64$. notamment chez le plus jeune, quand l'enquête alimentaire est faite auprès des parents.

Une étude cas-témoin, limitée, chez 32 enfants de 11 ans de poids normal ou en surpoids, n'a pas montré de différence d'apport lipidique entre les deux groupes mais, au contraire, un apport énergétique et glucidique plus élevédans le groupe poids normal. On peut évoquer une activité physique plus importante chez les enfants de poids normal, une sous-estimation chez les enfants en surpoids, le caractère dynamique de l'obésité (l'important est ce que l'enfant consommait en période de prise de poids), ou une susceptibilité génétique expliquant que les enfants plus forts mangent trop «pour eux » [33].

Dans une large étude française auprès de 646 enfants de 4 ans, les apports énergétiques des garçons en surpoids sont plus élevés (pas ceux de filles) que ceux des garçons ayant un poids normal, les apports lipidiques sont un peu plus élevés en valeur absolue, mais pas en pourcentage de l'apport énergétique total (AET) ; alors que, dans les deux sexes, l'activité physique est beaucoup plus faible chez les enfants en surpoids [34].

Une étude concernant I'alimentation et le poids des enfants et l'obésité parentale a montré une corrélation entre l'apport lipidique et la masse grasse, à nouveau chez les garçons mais pas chez les filles; et, pour l'ensemble, une corrélation entre apport lipidique des enfants et obésité maternelle, comme si les mères obèses étaient surtout nourricières pour les garçons [35].

Une autre étude, chez 181 enfants de 4 à 16 ans, n'a pas montré non plus de différence des apports lipidiques en pourcentage de l'AET entre enfants obèses et non obèses, mais un apport énergétique et lipidique en valeur absolue plus élevé chez les enfants obèses; mais dans cette étude, ceux-ci étaient non seulement un peu plus âgés mais un peu plus grands et bien sûr beaucoup plus lourds, de sorte que leurs dépenses étaient sans doute plus élevées malgré un score d'activité physique identique. Dans cette étude en modèle de régression multiple il existe une corrélation entre pourcentage de masse grasse et apport énergétique $(p<0,001)$ mais pas entre masse grasse et apport lipidique [36]. Ainsi la relation avec les lipides est très inconstante en ce qui concerne le pourcentage de lipides, et est plus fréquemment observée chez les garçons. Le rôle de l'apport énergétique global et des dépenses énergétiques semble l'emporter.

Puisque l'obésité est une affection dynamique on peut considérer que le poids dépend de ce que les sujets ont mangé avant ou au cours de la prise de poids. Ainsi une étude prospective concernant des enfants de 4 ans de poids nor- 


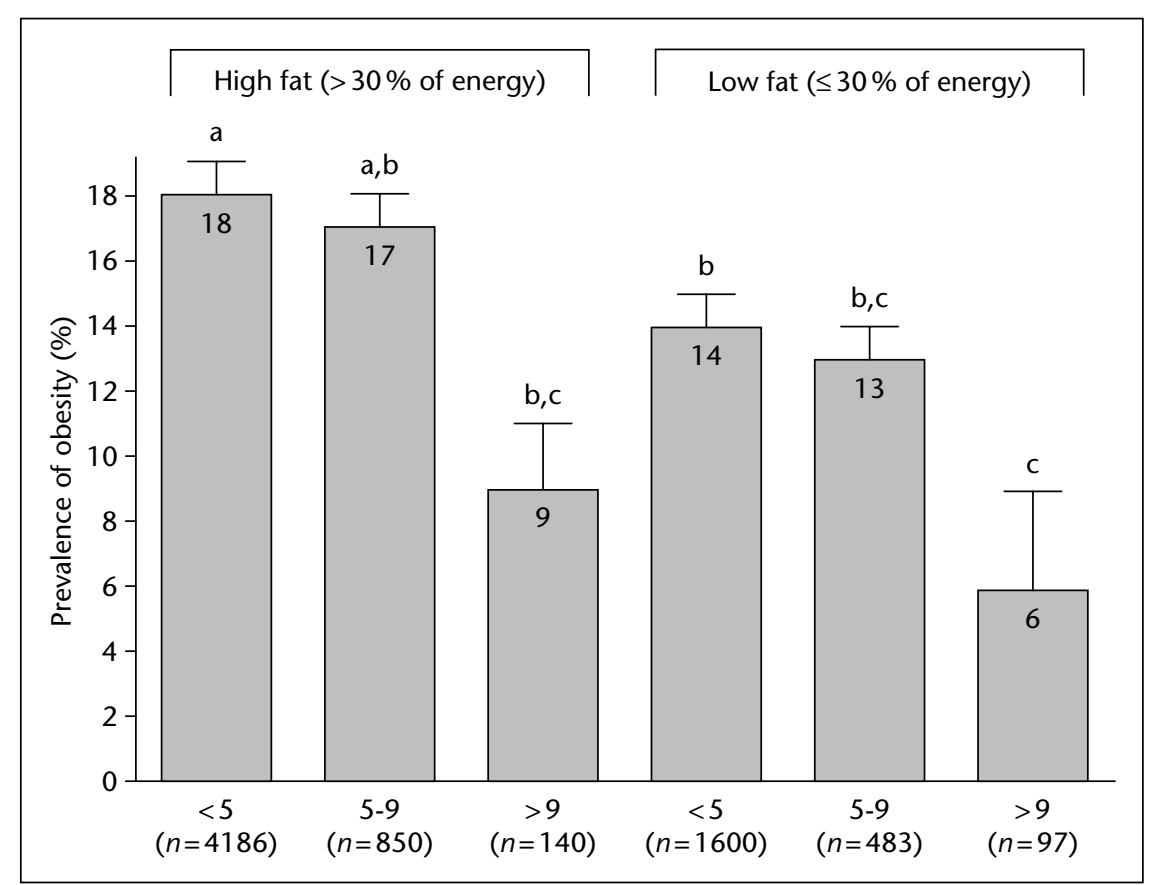

Figure 3. Un régime plus élevé en lipides n'est pas associé à une forte prévalence de l'obésité si l'apport en fruits et légumes est élevé. D'après Ledikwe, AJCN 2006 ; 83 : 1362-8.

mal a analysé les apports alimentaires des enfants avec au moins 5 enquêtes durant 6 ans : à l'âge de 10 ans, la comparaison entre ceux qui étaient du $3^{\mathrm{e}}$ tertile (poids élevé) et ceux qui étaient du $1^{\text {er }}$ tertile (poids normal) n'a pas montré de différence d'apports en énergie $\left(\mathrm{kcal} / \mathrm{jour} / \mathrm{m}^{2}\right)$, en lipides, en protides, ni de différence $d^{\prime}$ activité physique. Autrement dit, en dehors d'une hypothétique sousestimation, les enfants qui sont devenus plus forts $n^{\prime}$ ont pas mangé plus pendant les 6 années précédant leur prise de poids. II pourrait donc y avoir une susceptibilité individuelle forte chez les enfants prédisposés génétiquement, permettant d'affirmer qu'ils mangent trop « pour eux » mais pas plus que les autres [37] (tableau 1). Une autre étude prospective mesurant la densité énergétique (DE) chez des enfants au cours de 4 enquêtes alimentaires entre l'âge de 3 ans et 6 ans a montré une augmentation de la $D E$ avec l'âge mais aucune corrélation avec le poids, ni aucune différence selon la prédisposition familiale à l'obésité [38]. Dans une seconde publication récente sur la même population, les auteurs montrent qu'à cet âge les apports alimentaires sont inversement proportionnels à la densité énergétique, ce qui témoigne d'une adaptation (en dehors des boissons) avec toutefois une détérioration possible de cette compensation chez les enfants à haut risque d'obésité [39].

Chez 114 enfants de 6-8 ans vivant avec leurs parents biologiques [14 obèses, 100 de poids normal dont 50 à haut risque (un parent obèse) et 50 à bas risque (deux parents de poids normal)], une enquête alimentaire de 7 jours en double-pesée, la mesure de la dépense énergétique par eau doublement marquée, et la mesure de la masse grasse par dilution au deutérium ont été réalisées: les enfants obèses avaient des apports lipidiques plus élevés (pas de sous-évaluation, d'après le rapport apports énergétiques/dépenses énergétiques) que les enfants à bas risque. Ceux à haut risque ne consommaient pas plus d'énergie ou de lipides que ceux à bas risque. Par contre, pour l'ensemble de la population, il existait une corrélation quoique faible $(p<0,05)$ entre apport lipidique (en \%) et masse grasse. Ceci pourrait confirmer que les enfants à risque d'obésité génétique ne mangent pas plus que les autres mais, s'ils le font, et s'ils mangent davantage de lipides, leur risque de prendre du poids est bien sûr très important [40].

Le facteur génétique ou la réduction des dépenses sont donc des facteurs-clés dans la prise de poids, s'exprimant à la faveur de facteurs nutritionnels. Ainsi une étude longitudinale chez des enfants de 4 ans jusqu'à 11 ans a montré une augmentation de I'IMC avec le nombre d'heures de télévision : mais I'association d'une sédentarité élevée (heures de télévision) à une alimentation hyperlipidique dans cette étude a augmenté considérablement I'accroissement de l'IMC avec l'âge [41].

Cependant, le gain de poids ne semble pas dû à l'apport lipidique en tant que tel. Pearcey [42] a apparié des sujets de poids élevé (IMC: $30 \mathrm{~kg} / \mathrm{m}^{2}$ ) en poids stable ou en gain de poids. Seuls les apports énergétiques et glucidiques étaient plus élevés dans le groupe gain de poids, I'activité physique étant identique.

Une étude longitudinale a concerné 155 sujets adultes suivis 10 à 12 ans. Ceux qui ont pris plus de $5 \mathrm{~kg}$ avaient initialement les mêmes poids, IMC, masse grasse, masse maigre que ceux n'ayant pas pris plus de $5 \mathrm{~kg}$ au terme de ce suivi, mais ils avaient une diminution initiale de leur métabolisme de base, exprimé par kg de masse maigre [43] : ceci est sans doute lié à un facteur génétique qui s'exprimera à la faveur de facteurs nutritionnels, l'apport lipidique étant un bon candidat pour faire basculer la balance énergétique. Cette prédisposition génétique peut également être illustrée par

Tableau 1. Étude prospective enfants. D'après Remer, Eur J Clin Nutr 2002 ; 56 : 1046.

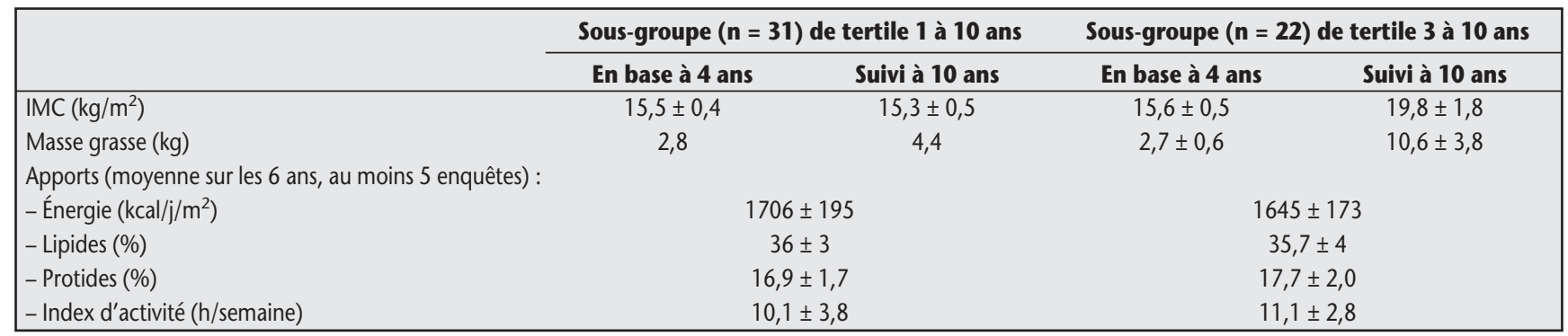


une autre étude : deux groupes d'hommes jeunes de poids normal, l'un ayant deux parents de poids normal, l'autre deux parents de poids élevé ont été comparés [44] : alors que l'IMC, la masse maigre, la glycémie, les lipides plasmatiques, les acides gras libres, la leptinémie des deux groupes (à jeun et en post-prandial) sont identiques; I'insulinémie, la résistance à I'insuline (HOMA), I'oxydation glucidique en post-prandial sont plus élevées, et l'oxydation lipidique en post-prandial est plus basse dans le groupe d'hommes jeunes ayant deux parents de poids élevé : ainsi, avant même le gain de poids, la génétique s'exprime à travers une moindre capacité à oxyder les lipides témoignant donc d'une capacité accrue à les stocker.

\section{Quel est l'impact de la réduction de l'apport lipidique?}

Ainsi les lipides jouent un rôle, dans la prise de poids, bien que non exclusif. Cependant la réduction de l'apport lipidique est loin de faire l'unanimité dans la prévention de l'obésité et dans la prise en charge de celle-ci [23, 28, 45]. En termes de prise en charge de l'obésité, on sait que toute réduction d'apports énergétiques est assortie d'une résistance à l'amaigrissement dont on sait qu'elle est à la fois due à des facteurs métaboliques et à un échappement diététique [46]. Les études cliniques d'intervention avec une réduction de l'apport lipidique montrent d'ailleurs une faible corrélation entre réduction du pourcentage de lipides et perte de poids [23, 28]. D'autre part, on sait qu'une perte de poids intentionnelle ne réduit pas toujours la mortalité associée à l'obésité. Ceci peut être en partie dû à la perte des effets bénéfiques de la graisse sous-cutanée périphérique et de la masse maigre [47]. De plus, il a été montré récemment que le pronostic cardiovasculaire et la mortalité sont moindres chez les sujets coronariens ayant un excès de poids ou une obésité modérée que chez des sujets plus minces $[48,49]$. Ceci pourrait être dû au caractère non-discriminant de I'IMC et à la non-prise en compte de la masse maigre et de l'obésité abdomino-viscérale $[49,50]$. On peut aussi évoquer les effets délétères de la réduction lipidique ou d'autres mesures diététiques sans perte de poids associée.

Ainsi, dans un objectif de prévention, l'étude WHI (Women's Health Initiative) a recommandé ou non une réduction d'apport lipidique, (de façon randomisée), dans une population de 48835 femmes de 62,3 ans, en moyenne, suivies 8,1 ans [51]. Partant de $37,8 \%$ de lipides dans I'AET, I'objectif était d'atteindre un apport lipidique de $20 \%$. Dans le groupe actif, l'apport observé a atteint
$28,8 \%$, et il est resté à $37,8 \%$ dans le groupe témoin. Il était en outre recommandé une augmentation de l'apport en céréales, en fruits et en légumes. Une perte de poids maximum a été obtenue au bout d'un an $(-2,2 \mathrm{~kg})$ puis le poids a rejoint progressivement le groupe témoin au bout de 4 à 6 ans, quels que soient l'âge initial ou l'IMC initial ou l'ethnie. II est remarquable de noter que dans les deux groupes la perte de poids était proportionnelle à la réduction de l'apport lipidique, mais modeste, atteignant 1,25 à $1,50 \mathrm{~kg}$ pour une réduction de plus de $11 \%$ de l'apport lipidique [51]. La réduction de l'incidence du cancer du sein (objectif principal) a été non significative (-9\%) sauf pour le groupe partant des apports lipidiques les plus élevés (en pourcentage) [52]. Chez les femmes ayant déjà un cancer du sein, l'étude récente WHEL [53] n'a pas montré de bénéfices d'une telle diététique hypolipidique. Mais dans l'étude WHI, aucune différence n'a été obtenue en termes de réduction des événements coronariens, des accidents vasculaires cérébraux et des maladies cardiovasculaires [54]. Cependant, dans les groupes ayant atteint les apports les plus bas en acides gras saturés et en acides gras trans, une réduction de $19 \%$ et de $16 \%$ de l'incidence des cardiopathies ischémiques a été observée. $A$ contrario celles ayant des antécédents de maladie cardiovasculaire $(3,4 \%$ de la population) ont eu une augmentation des événements cardiovasculaires [54-56]. En réalité la réduction pondérale a été modeste et non durable [51], et elle peut donc être considérée comme insuffisante.

Par contre, chez des obèses ayant bénéficié $d^{\prime}$ une chirurgie bariatrique générant une perte de poids de $22 \mathrm{~kg}$, il a été observé une moindre progression de l'athérosclérose carotidienne que chez des obèses traités par régime [57], identique à celle de sujets de poids normal.

L'intérêt d'un faible apport lipidique chez des sujets porteurs d'une athérosclérose coronarienne doit cependant être discuté : dans une cohorte de 235 femmes ménopausées porteuses d'une maladie coronarienne avec au moins une sténose à plus de $30 \%$ et sans traitement hormonal substitutif, suivies 3 ans, 2243 segments coronariens ont été analysés. Classés par quintiles d'apports en acides gras saturés, les femmes ayant les apports les plus bas en lipides (et en acides gras saturés) ont eu une plus grande progression de leur athérosclérose [58]. Or il a été établi par Lefebvre [59] qu'un régime hypolipidique standard (STEP I à $28 \%$ de lipides et STEP II à $24 \%$ ) et pauvre en acides gras saturés entraînait, chez des hommes maintenant stable leur poids durant l'étude, certes une réduction du cholestérol LDL de 6,8\% et de $11,7 \%$ respectivement, mais aussi une réduction du cholestérol $\mathrm{HDL}$ de $7,5 \%$ et de $11,2 \%$, ainsi que l'augmentation des triglycé- rides de $14,3 \%$ et de $16,2 \%$, ce qui correspond au profil athérogène de la dyslipidémie du syndrome métabolique et du diabète ; la réduction du cholestérol LDL était d'autant plus faible que les sujets avaient une insulinorésistance. De plus, l'on sait depuis les travaux de Dreon $[60,61]$ que la réduction de l'apport lipidique est assortie d'une augmentation de I'incidence du phénotype B des LDL, associé à des $L D L$ petites et denses, la taille des LDL étant inversement corrélée aux triglycérides et à l'augmentation des hydrates de carbone (ellemême inversement corrélée au pourcentage de l'apport lipidique dans ces études).

La réduction de l'apport lipidique est donc peu favorable en l'absence de perte de poids consécutive ou associée chez les sujets ayant un syndrome métabolique, une dyslipidémie mixte, un diabète ou une athérosclérose, en partie parce qu'elle s'accompagne inévitablement d'un apport glucidique accru [62]. Dans une étude plus récente encore, Krauss [63] a montré qu'en poids stable un régime hypolipidique entraîne un moins bon profil lipidique (plus athérogène) qu'un régime hyperlipidique avec un apport en acides gras saturés modéré ; mais après perte de poids un régime hyperlipidique induit un moins bon profil lipidique (excepté la taille des LDL plus grandes, et $a$ priori plus favorable) [64]. En cas de régime hyperlipidique, le niveau d'apport en acides gras saturés ne modifie pas le profil lipidique. Le phénotype $B$ des LDL était corrélé à l'apport en hydrates de carbone mais beaucoup moins fortement après perte de poids [63] (figure 4). Ainsi, il apparaît que, plus que la réduction des lipides, c'est la réduction des calories glucidiques et lipidiques et donc des apports énergétiques dans leur ensemble qui importe et, plus encore, c'est la réduction du poids qui l'emporte, sans doute à la faveur d'un accroissement des dépenses énergétiques.

D'ailleurs, l'étude WHLP (Women's Healthy Lifestyle Project) a randomisé deux groupes de femmes préménopausées de 47 ans et ayant un IMC de $25 \mathrm{~kg} / \mathrm{m}^{2}$ en moyenne. Afin de prévenir le gain de poids de la ménopause, le groupe intervention a reçu des consignes pour réduire son apport en lipides et pour accroître son activité physique : au bout de 54 mois le poids n'a pas augmenté dans le groupe intervention alors qu'il a augmenté de $2,4 \mathrm{~kg}$ dans le groupe témoin, et les facteurs de risque étaient meilleurs avec ou sans traitement hormonal substitutif [65] ; ceci était associé à une moindre progression de l'athérosclérose carotidienne [66].

Enfin au-delà de I'aspect quantitatif I'apport qualitatif est sans doute déterminant tant sur le plan de la nature des lipides (importance de l'apport en acides gras mono-insaturés et des acides gras oméga 3), que de la nature des 


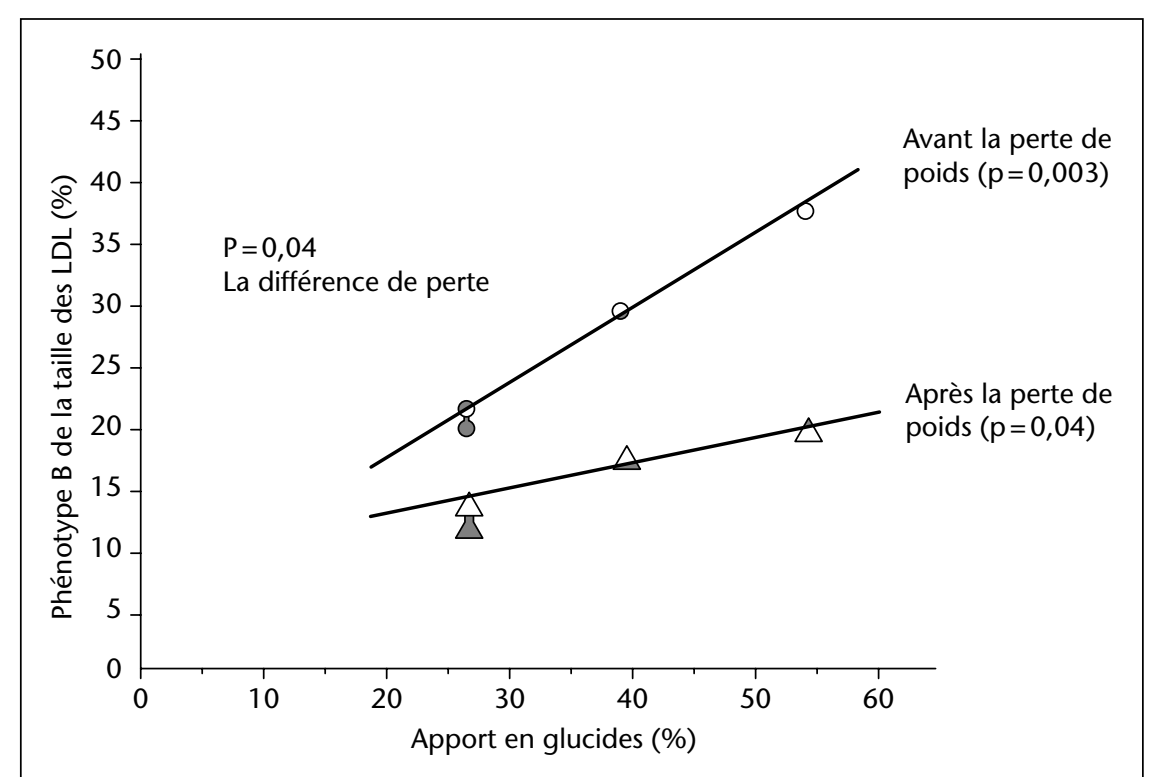

Figure 4. Le phénotype B DES LDL (petites et denses) est corrélé à l'apport en hydrates de carbone moins fortement après la perte de poids. D'après Krauss. AJCN 2006 ; 83 : 1025-31.

glucides (rôle de l'index gycémique bas), mais c'est un autre sujet... À titre d'exemple, une étude a montré que l'adjonction d'acides gras oméga 3 à longues chaînes $(4,2 \mathrm{~g} / \mathrm{j})$ dans un programme de perte de poids obtenue par réduction de l'apport lipidique chez des femmes en surpoids insulino-résistantes ajoute, au bout de 24 semaines, aux effets de la perte de poids (insulino-sensibilité accrue, baisse des triglycérides et diminution de l'inflammation) une réduction plus importante des triglycérides et une augmentation de l'adiponectine [67].

\section{Conclusion}

Les lipides ont d'un point de vue métabolique les qualités et propriétés requises pour être de très bons candidats pour favoriser la prise de poids: de ce point de vue, ils peuvent non seulement contribuer fortement à positiver la balance énergétique mais aussi à favoriser la lipogenèse. Toutefois les études expérimentales, épidémiologiques et cliniques montrent qu'ils ne sont pas des facteurs exclusifs et ne sont ni nécessaires ni suffisants. Le gain de poids est lié au fait d'avoir de façon régulière des apports énergétiques supérieurs aux dépenses: si les lipides peuvent y contribuer, les calories ingérées (et la densité énergétique) et dépensées globalement importent plus, ainsi que la susceptibilité individuelle (liée à des facteurs génétiques).

Dans la genèse de l'obésité, l'apport lipidique total peut jouer un rôle important mais il n'est pas la cause de l'épidémie d'obésité, qui est multifactorielle.
La prévention de l'obésité passe par une lutte contre la sédentarité et une réduction de la densité énergétique de l'alimentation à laquelle les lipides peuvent contribuer fortement. La réduction de l'apport lipidique isolément $n^{\prime}$ est pas une mesure efficace dans la prévention du gain de poids : c'est la réduction de l'apport énergétique et l'accroissement des dépenses qui importent.

Dans la prise en charge de l'obésité, il faut mettre en œuvre les mesures permettant une réduction de la graisse abdomino-viscérale et le maintien de la masse maigre.

Chez les sujets ayant une insulino-résistance et/ou une athérosclérose, la réduction des lipides sans perte de poids entraine une augmentation du risque cardiovasculaire : il faut sans doute privilégier la réduction des glucides, accroître l'activité physique, améliorer la qualité des lipides et des apports glucidiques et accroître les nutriments protecteurs ; augmenter enfin l'activité physique afin de maintenir la masse maigre.

\section{RÉFÉRENCES}

1. SCHRAUWEN P, VAN MARKEN LICHTENBELT WD, SARIS WH, WESTERTERP KR. Changes in fat oxidation in response to a high fat diet. Am J Clin Nutr 1997 ; 66 : 276-82.

2. PRENTICE AM, DUNN MRC. Manipulation of dietary fat and energy density and subsequent effects on substrate flux and food intake. Am J Clin Nutr 1998 ; 67(Suppl) : 535S-541S.

3. HALL KD, BAIN HL, CHOW CC. How adaptations of substrate utilization regulate body composition. Int / Obes 2007 ; 31 : 1378-83.
4. JEBB SA, PRENTICE A, GOLDBERG GR, MURGATROYD PR, BLACK AE, COWARD WA. Changes in macronutrient balance during over and underfeeding assessed by $12-\mathrm{d}$ continuous whole-body calorimetry. Am / Clin Nutr 1996 ; 64 : 259-66.

5. RAVUSSIN E. Metabolic differences and the development of obesity. Metabolism 1995; 44(Suppl 3) : 12-4.

6. SCHUTZ Y, TREMBLAY A, WEINSIER RL, NELSON KM. Role of fat oxidation in the long-term stabilization of body weight in obese women. Am J Clin Nutr 1992 ; 55 : 670-4.

7. ASTRUPA, BUEMANN B, CHRISTENSEN N, TOUBRO $S$. Failure to increase lipid oxidation in response to increasing dietary fat in formerly obese women. Am J Physiol 1994 ; 266 : E592E599.

8. HEITMANN BI, LISSNER L, SORENSEN TIA, ET AL. Dietary fat intake and weight gain in women with genetic predisposition. Am / Clin Nutr $1995 ; 61$ : 1213-7.

9. LARSON DE, FERRARO RT, ROBERTSON DS, RAVUSSIN E. Energy metabolism in weight stable post-obese individuals. Am / Clin Nutr $1995 ; 62$ : 735-9.

10. BELLEVILLE J. Le goût pour les lipides. Cah Nutr Diet $1998 ; 33$ : 365-72.

11. DREWNOWSKI A, HANN C. Food preferences and reported frequencies of food consumption as predictors of current diet in young women. Am J Clin Nutr $1999 ; 70:$ 28-36.

12. SALBE A, DEL PARIGI A, PRATLEY RF, DREWNOWSKI A, TATARANNI PA. Taste preferences and body weight in an obesity-prone populations. Am / Clin Nutr 2004 ; 79 : 372-8.

13. LECERF JM, EVRARD A, FRESSIN C. Dietary preference quoted by obese and non obese children are not quite different. Int / Obes 1998 ; 22 : \$21.

14. DREWNOWSKI A, KURTH CL, RAHAIN JE. Taste preferences in human obesity : environmental and familial factors. Am / Clin Nutr 1991 ; 54 : 635-41.

15. BeLl eA, CAstellanos VH, PELKMAN CL, THURWART ML, ROLL BJ. Energy density of foods affects energy intake in normal weight women. Am / Clin Nutr 1998 ; 67 : 412-20LL.

16. LARSON DE, TATARANNI PA, FERRARO RT, RAVUSSIN E. Ad libitum food intake on a "cafeteria diet" in Native America women : relations with body composition and 24h energy expenditure. Am J Clin Nutr $1995 ; 62$ : 911-7.

17. DREWNOWSKIA, ALMIRON-ROIG E, MARMONIER C, LLUCH A. Dietary energy density and body weight : is there a relationship. Nutr Rev 2004; 62 : 403-13.

18. SHEPARD TY, WEIL KM, SHARP TA, ETAL. Occasional physical inactivity combined with a high-fat diet may be important in the development and maintenance of obesity in human subjects. Am / Clin Nutr 2001 ; 73 : 703-8. 
19. LAMMERT O, FABER P, GRUNNET N, ET AL. Overconsumption of carbohydrate or fat in young men: effects on energy expenditure during sleep and faecal energy loss. Int / Obes $1998 ; 22$ : S3-S184.

20. WESTERTERP MS, WILSON SAJ, ROLLAND V. Diet induced thermogenesis measured over 24 $\mathrm{h}$ in a respiration chamber : effect of diet composition. Int / Obes $1999 ; 23$ : 283-92.

21. BABA NH, SULTAN R, CORTAS N, HABBAL Z. Diet composition affects weight gain, adiposity and blood parameters in healthy human volunteers. Nutr Res 1999 ; 19 : 1313-26.

22. LISSNER L. Heitmann. Dietary fat and obesity : evidence from epidemiology. Eur / Clin Nutr $1995 ; 49$ : 79-90.

23. BRAY GA, POPKIN BP. Dietary fat intake does affect obesity. Am / Clin Nutr 1998 ; 68 : 1157 73.

24. BOLTON-SMITH C, WOODWARD M. Dietary composition and fat to sugar ratios in relation to obesity. Int J Obes $1994 ; 18: 820-8$.

25. GIBNEY MJ. Dietary guidelines : a critical appraisal. J Hum Nutr Diet 1990 ; 3 : 245-54.

26. MAC DIARMID JL, VAID A, CADE JE, BLUNDEL$L J E$. The sugar fat relationship revisited : differences in consumption between men and women of varying BMI. Int / Obes 1998 ; 22 : 1053-61.

27. HEINI AF, WEINSIER RL. Divergent trends in obesity and fat intake patterns; The American paradox. Am / Med 1997 ; 102 : 259-64.

28. WILLETT WC. Is dietary fat a major determinant of body fat? Am / Clin Nutr 1998 ; 67(Suppl) : 556S-562S

29. PRENTICE AM, JEBB SA. Obesity in Britain : gluttony or sloth? BMJ $1995 ; 311$ : 437-9.

30. TOGO P, OSLER M, SORENSEN TA, HERMAN$\mathrm{NBL}$. Food intake patterns and body mass index in observational studies. Int J Obes 2001 ; $25: 1741-51$

31. LE DIKWE JH, BLANCK HM, KHAN LK, SERDULA MK. Dietary energy density is associated with energy intake and weight status in US adults. Am / Clin Nutr 2006 ; 83 : 1362-8.

32. FIELD AE, WILLETT WC, LISSNER L, COLDITZ GA. Dietary fat and weight gain among women in the Nurses' Health Study. Obesity $2007 ; 15$ : 967-76.

33. ROCANDIO AM, ANSOTTEOUI L, ARROYO M. Comparison of dietary intake among overweight and non overweight schoolchildren. Int J Obes $2001 ; 25$ : 1651-5.

34. JOURET B, AHLUWALIA N, CRISTINI C, ET AL. Factors associated with overweight in preschool-age children in southwestern France. Am / Clin Nutr 2007 ; 85 : 1643-9.

35. NGUYEN VT, LARSON DE, JOHNSON RK, GORAN MI. Fat intake and adiposity in children of lean and obese parents. Am / Clin Nutr 1996 ; $63: 507-13$.
36. GILLIS LJ, KENNEDY LC, GILLIS AM, BAR OR O. Relationship between juvenile obesity, dietary energy and fat intake and physical activity. Int J Obes $2002 ; 26: 458-63$.

37. REMER T, DIMITRIOU T, KERSTING M. Does fat intake explain fitness in healthy children? Eur J Clin Nutr $2002 ; 56$ : 1046-7.

38. KRAL TVE, BERKOWITZ RI, STUNKARD AJ, STALLING VA, BROWN DD, FAITH MS. Dietary energy density increases during early childhood irrespective of familial predisposition to obesity: results from a prospective cohort study. Int J Obes 2007 ; 31 : 1061-7.

39. KRAL TVF, STUNKARD AJ, BERKOWITZ RI, STALLING VA, BROWN DD, FAITH MS. Daily food intake in relation to dietary energy density in the free-living environment: a perspective analysis of children born at different risk of obesity. Am J Clin Nutr 2007 ; 86 : 41-7.

40. MCGLOIN AF, LIVINGSTONE MB, GREENE LC, WEBB SE. Energy and fat intake in obese and lean children at varying risk of obesity. Int I Obes $2002 ; 26: 200-7$.

41. PROCTOR MH, MOORE LL, GAO D, ET AL. Television viewing and change in body fat from preschool to early adolescence: The Framingham Children's Study FREE. Int / Obes 2003 ; 27 : 827-33.

42. PEARCEY SM, DE CASTRO JM. Food intake and meal patterns of weight stable and weightgaining persons. Am / Clin Nutr 2002; 76 : 107-12.

43. BUSCEMI S, VERGA S, CAIMI G, CERASOLA G. Low relative resting metabolic rate and body weight gain in adult Caucasian Italians. Int $)$ Obes $2005 ; 29$ : 287-91

44. GIACCO R, CLEMENTE G, BUSIELLO L, ET AL. Insulin sensitivity is increased and fat oxidation after a high-fat meal is reduced in normalweight healthy men with strong familial predisposition to overweight. Int / Obes 2003 ; 27 : 790-6.

45. ASTRUP A. The role of dietary fat in the prevention and treatment of obesity. Efficacy and safety of low-fat diets. Int / Obes 2001; 25(Suppl1) : S46-S50.

46. HEYMSFIELD SB, HARP JB, REITMAN ML, ET AL. Why do obese patients not lose more weight when treated with low-calorie diets? A mechanistic perspective. Am / Clin Nutr 2007; 85 : 346-54.

47. BERENTZEN T, SOBRENSEN TIA. Effects of intended weight loss on morbidity and mortality : possible explanations of controversial studies. Nutr Rev 2006 ; 64 : 502-7.

48. GALAL W, VAN DOMBURG RT, FERONGA$\mathrm{HHH}$, ET AL. Relation of body mass index to outcome in patients with known or suspected coronary artery disease. Am / Cardiol 2007 ; 99 : 1485-90.
49. ROMERO-CORRAL A, MONTORI VH, KORINEF JK, ET AL. Association of body weight with total mortality and with cardiovascular events in coronary artery disease : a systematic review of cohort studies. Lancet 2006 ; 368 : 666-78.

50. WANNA METHEE SG, SHAPER AG, LENNON L, WHINCUP PH. Decreased muscle mass and increased central adiposity are independently related to mortality in older men. Am / Clin Nutr $2007 ; 86: 1339-46$

51. HOWARD BV, HANSON SE, STEPHANICK HL, ET AL. Low fat dietary pattern and weight change over 7 years. The Women's Health initiative dietary modification trial. JAMA 2006 ; 295 : 39-49.

52. PRENTICE RL, CAAN B, CHLEBOWSKI RT, ET AL. Low fat dietary pattern and risk of invasive beast cancer. The Women's Health Initiative randomised controlled dietary modification trial. JAMA 2006 ; 295 : 629-42.

53. PIERCE JP, NATARATAN L, CAAN BJ, ET AL. Influence of a diet very high in vegetables, fruit and fiber and low in fat on prognosis following treatment for breast cancer. JAMA 2007 ; 298 : 289-98.

54. HOWARD BV, VAN HORN LH, SIA H, ET AL. Low-fat dietary pattern and risk of cardiovascular disease. The Women's Health Initiative randomized controlled dietary modification trial. JAMA 2006 ; 296 : 655-66.

55. HOWARD BV. Dietary fat and cardiovascular disease : putting the women's Health Initiative in perspective. Nutr Metab Cardiovasc Dis $2007 ; 17: 171-4$.

56. ANDERSON CAM, APPEL LJ. Dietary modification and CVD prevention. JAMA 2006; 295 : 693-5.

57. KARASON K, WIKSTRAND ], SJOSTROM L, WENDELHAG I. Weight loss and progression of early atherosclerosis in the carotid artery : a four-year controlled study of obese subjects. Int J Obes $1999 ; 23$ : 948-56.

58. MOZAFFARIAN D, RIMM EB, HERRINGTON DM. Dietary fats, carbohydrate, and progression of coronary atherosclerosis in postmenopausal women. Am J Clin Nutr 2004; 80 : 1175-84.

59. LEFEBVRE M, CHAMPAGNE CM, TULLEY RT, ROOT JC, MOST MM. Individual variability in cardiovascular disease risk factor responses to low-fat and low-saturated-fat diet in men: body mass index, adiposity, and insulin resistance predict changes in LDL cholesterol. Am J Clin Nutr $2005 ; 82$ : 957-63.

60. DREON DH, FERNSTROM HA, WILLIAM PT, KRAUSS RM. Reduced LDL particle size in children consuming a very-low-fat diet is related to parental LDL -subclass patterns. Am / Clin Nutr $2000 ; 71: 1611-6$ 
61. DREON DM, FERNSTROM HA, WILLIAM PT, KRAUSS RM. A very-low-fat diet is not associated with improved lipoprotein profile in men with a predominance of large, low-density lipoproteins. Am / Clin Nutr 1999; 69 : 411-8.

62. KATAN MB. Alternative to low-fat-diet. Am / Clin Nutr $2006 ; 83: 889-90$.

63. KRAUSS RM, BLANCHE PJ, RAWLINCS RS, FERNSTROM HS, WILLIAM PT. Separate effects of reduced carbohydrate intake and weight loss on atherogenic dyslipidemia. Am / Clin Nutr $2006 ; 83: 1025-31$.
64. WOOD RT. Effect of dietary carbohydrate restriction with and without weight loss on atherogenic dyslipidemia. Nutr Rev 2006 ; 64 : 539-45.

65. KULLER LH, SIMKIN-SILVERMAN LR, WING PR, MEILAHN EN, IVES DG. Women's Healthy Lifestyle Project : a randomized clinical trial. Results at 54 months. Circulation $2001 ; 103: 32-7$.

66. WILDMAN RP, SCHOTT LL, BROKWELL S, KUKKER LH, SUTTON-TYRRELL K. A dietary and exercise intervention slows menopause- associated progression of subclinical atherosclerosis as measured by intima-media thickness of the carotid arteries. J Am Coll Cardiol 2004 ; 44 : 579-85.

67. KREPS JD, BROWNING LM, MC LEAN NK, ET AL. Additive benefits of long-chain n-3 polyunsaturated fatty acids and weight loss in the management of cardiovascular disease risk on overweight hyperinsulinemic women. Int I Obes $2006 ; 30$ : 1535-44. 